\title{
Trapping and Driving Individual Charged Micro-particles in Fluid with an Electrostatic Device
}

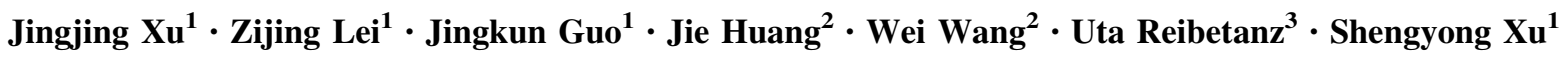

Received: 7 December 2015/Accepted: 5 February 2016/Published online: 10 March 2016

(C) The Author(s) 2016. This article is published with open access at Springerlink.com

\begin{abstract}
A variety of micro-tweezers techniques, such as optical tweezers, magnetic tweezers, and dielectrophoresis technique, have been applied intensively in precise characterization of micro/nanoparticles and bio-molecules. They have contributed remarkably in better understanding of working mechanisms of individual sub-cell organelles, proteins, and DNA. In this paper, we present a controllable electrostatic device embedded in a microchannel, which is capable of driving, trapping, and releasing charged micro-particles suspended in microfluid, demonstrating the basic concepts of electrostatic tweezers. Such a device is scalable to smaller size and offers an alternative to currently used micro-tweezers for application in sorting, selecting, manipulating, and analyzing individual micro/nanoparticles. Furthermore, the system offers the potential in being combined with dielectrophoresis and other techniques to create hybrid micro-manipulation systems.
\end{abstract}

Keywords Electrostatic tweezers $\cdot$ Charged particles $\cdot$ Coulomb potential well $\cdot$ Manipulation $\cdot$ Trap

\section{Introduction}

In the past two decades, characterization and application of micro/nanoparticles have attracted much attention. In this field, micro-tweezers have played an important role in capturing and manipulating micro/nanoscale particles, molecules, and atoms. For instance, optical tweezers are

Electronic supplementary material The online version of this article (doi:10.1007/s40820-016-0087-3) contains supplementary material, which is available to authorized users.

Wei Wang

ww@pku.edu.cn

Shengyong Xu

xusy@pku.edu.cn

1 Key Laboratory for the Physics \& Chemistry of Nanodevices, and Department of Electronics, Peking University, Beijing 100871, People's Republic of China

2 Institute of Microelectronics, Peking University, Beijing 100871, People's Republic of China

3 Medical Faculty, Institute for Medical Physics and Biophysics, University of Leipzig, 04103 Leipzig, Germany using one or multiple laser beams to create a gradient field towards the center of the laser focal point, offering a unique way to trap micro/nanoparticles. Furthermore, it has been shown that atoms can be trapped and cooled in studies on Bose-Einstein condensation [1-3], atomic clocks, and quantum phenomena [4-7]. Optical tweezers have also been extensively applied in a variety of studies on microparticles [8-10], cells [10], bacteria [11], sub-cell organelles, bio-macromolecules [12, 13], and nanoparticles [14, 15]. Another kind of effective tweezers based on dielectrophoresis (DEP) technique has been applied in sorting [16-19], trapping [20], characterizing [17], and transporting [20] dielectric particles, cells, and bio-macromolecules by polarizing and driving them in non-uniform electric fields $[21,22]$. The "Paul Trap," which utilizes high-frequency alternating electric fields on four specially designed electrodes, is capable of capturing charged particles [23, 24]. Magnetic tweezers, which utilize gradient magnetic fields as the trapping force, can shift and rotate a magnetic particle or other targeted objects modified with a magnetic bead [25-29]. This type of tweezers has been successfully applied in studying the role of topoisomerase in unwinding of DNA [30]. The concepts of electron tweezers which 
utilize a focused electron beam in a transmission electron microscope (TEM) for trapping and manipulating quantum dots and nanoparticles have also been addressed [31-33]. In addition, optofluidic systems are getting more and more attention in the field. Kayani et al. summarized not only the manipulation forces in controlling particles, but also the applications of optofluidics incorporating controlled particles in manipulating, sensing, and analyzing micro-particles and even bio-molecules nowadays and in the future [34]. There are also other related techniques applied in complex biosensing [35] and biological measurements [36]. All manipulation technologies mentioned above operate mainly on the basis of electromagnetic interaction. In fact, at the micro/nanoscales, the electromagnetic interaction is the only force work effectively among the known four fundamental forces in physics.

In live biosystems, a huge number of effective, rapid, and regular interactions occur every second among intracellular micro/nanoparticles and macromolecules, such as handling, transfer, specific binding, and catalytic reaction [37-43]. Logically, it is convincing that certain ordered manipulation and interaction mechanisms, rather than thermal motion alone, exist in these intracellular activities. However, in live biosystems, there is no essential condition for artificial optical tweezers, magnetic tweezers, DEP, or "Paul traps," which all need additional power supplies. Computational simulations have shown that the specific interaction between charged particles and local electric field contributed by charge distribution plays the key role in the complex but effective biological processes [44-51]. This is consistent with experimental evidences, which demonstrate that molecules (such as enzymes, DNA, proteins, etc.) in cells usually carry net charges, or even a specific charge distribution [50-56]. These facts lead to a new concept of tweezers, the electrostatic tweezers (EST), which work passively and do not require manmade powers, therefore they could exist in a living biosystem. In a study on the interaction between sunitinib and its specific target protein, Malinska et al. have found that the electrostatic interaction is a major factor, and sunitinib can adjust its conformation to fit the binding pocket as to enhance the electrostatic interactions [57].

As bio-macromolecules are in nanoscale, it is hard to observe the working status of the proposed natural ESTs or traps in a living cell directly. The first report on a nanosized electrostatic trap was reported in a TEM study of cadmium selenide $(\mathrm{CdSe})$ quantum dots, where a CdSe nanocrystal was observed floating and rotating over a carbon film [31]. In this work, the concept and feasible constructions of onedimensional (1D), 2D, and 3D electrostatic traps were systematically discussed. The first manmade electrostatic trap was reported by Krishnan and co-workers [58]. They have found that by utilizing net electrostatic charges unintentionally left in a nanoscale slit of a microfluidic channel, which resulted in a Coulomb potential, the device was capable of trapping gold nanoparticles and polymer beads. However, this kind of trap cannot capture particles selectively in a controllable way, or release them when needed. To the best of our knowledge, practical electrostatic manipulation devices with functions of driving, trapping, and releasing charged particles have not been reported.

In the present work, we attempted to demonstrate the basic concepts of EST: capture, manipulation, and release of charged particles using a controllable static Coulomb potential well. The electrostatic potential traps used in this work were formed by electrostatic charges distributed on specially designed metallic electrodes. Instead of using naturally formed electrostatic charges, the surface charge density was adjusted with a small external DC voltage source, making the resulting Coulomb force controllable. The limited height of the microfluidic channel of our devices supported the Coulomb potential wells in firmly trapping charged micro-particles. Additional electrodes were also used to apply an external electric field for driving and manipulating particles in the fluidic channels. Recorded with a video camera, motions of confined particles and clusters inside the Coulomb wells showed that these electrostatic devices work well as designed.

\section{Experimental}

\subsection{Simulation of the Electrostatic Coulomb Potential Trap}

Basically, when a metallic thin-film strip is connected to a static voltage power supplier, its surface will be covered with a certain amount of static charges so as to maintain a constant potential. A prototype device with three parallel metallic stripe electrodes was designed to show the basic features of an electrostatic Coulomb well. Figure 1 illustrates the Coulomb potential in an $x-y$ plane and at a constant distance to the 3 -stripe electrodes. Figure 1a shows the schematic structure of the 3-stripe electrodes, viewing in $y$ direction, where the electrodes have a width of $w$ and a length of $L$, and the spacing for the outer two electrodes is $d$. The definition for $x, y$, and $z$ coordinates is presented at the upper right corner. With suitable choices of the voltages applied to the stripes, and at suitable distance of $z$, the deep potential well is formed in the $x-y$ plane.

Figure $1 \mathrm{~b}$ plots a typical electrostatic Coulomb potential distribution in vacuum in $x-y$ plane at $z=15 \mu \mathrm{m}$, for a 3-stripe trap with $w=10 \mu \mathrm{m}, \quad d=50 \mu \mathrm{m} \quad$ and $L=100 \mu \mathrm{m}$, where a voltage of $2 \mathrm{~V}$ is applied between the positively outer stripes and the earthed central stripe. 


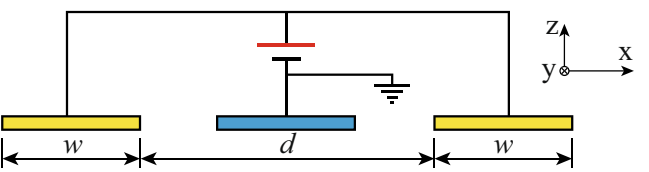

(a)

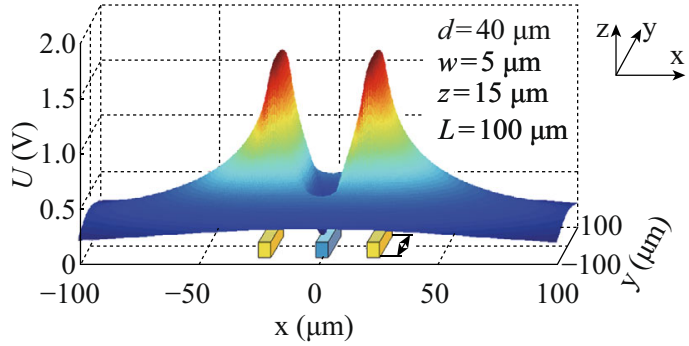

(b)

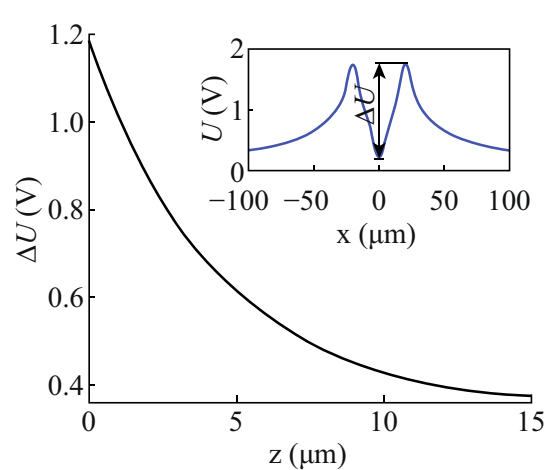

(c)

Fig. 1 Design and simulation of a 3-stripe electrostatic trap. a The schematic structure of the 3-stripe metal electrodes, viewing in $y$ direction. b Simulation result of electrostatic Coulomb potential in vacuum in the $x-y$ plane for 3-stripe trap at an excitation voltage of $2 \mathrm{~V}$, at $z=15 \mu \mathrm{m}$. c The curve of the potential depth $\Delta U$ at $z$ direction due to screen effect. The inset at the up right corner defines the potential depth $\Delta U$

The simulation of the Coulomb well is accomplished by MATLAB 2012b, and here successive over-relaxation method (SOR) is applicative to solve Laplace equation:

$\frac{\partial^{2} U}{\partial x^{2}}=\frac{\partial^{2} U^{2}}{\partial y^{2}}=\frac{\partial U}{\partial z^{2}}=0$

with the boundary conditions that $U=2 \mathrm{~V}$ on the outer two electrodes, while $U=0$ on the middle electrode and in the infinite. Considering the width $(w)$ and length $(L)$ of the electrode, the boundary conditions are expressed in the following form, respectively:

$$
\begin{aligned}
& U\left(-\left(\frac{1}{2} d+w\right)<x<-\frac{1}{2} d, \quad-\frac{1}{2} L<y<\frac{1}{2} L, \quad z=0\right) \\
& \quad=U\left(\frac{1}{2} d<x<\frac{1}{2} d+w, \quad-\frac{1}{2} L<y<\frac{1}{2} L, \quad z=0\right) \\
& \quad=2
\end{aligned}
$$$$
U\left(-\frac{1}{2} w<x<\frac{1}{2} w, \quad-\frac{1}{2} L<y<\frac{1}{2} L, \quad z=0\right)=0
$$

$U_{\infty}=0$.

In fact, the simulation results in Fig. 1 are based on simplified models, therefore they are qualitative analysis, and give a visible picture for the potential well used in this work. The situation for a real device under test is much more complicated. We have discussed some of the effects in Sect. 4, such as Brownian motion, screen effect, electroosmosis, and thermophoresis.

Such a trap is suitable for capturing positively charged objects. Generally, the depth of the trap, either in the potential $(U)$ or in energy (i.e. $q U$, assuming that the charged particle has a charge $q$ ), decreases rapidly with increasing height (in $z$ direction). When the screen effect is taken into account, the trap depth becomes even smaller than the stimulated values (Fig. 1b) in vacuum, because the screen effect remarkably reduces the effective surface charge density at the trap electrodes. It is stimulated by COMSOL $4.3 \mathrm{~b}$ that the potential depth $\Delta U$ at $z$ values (Fig. 1a) of $0,5,10$, and $15 \mu \mathrm{m}$ are $1.18,0.61,0.43$, and $0.38 \mathrm{~V}$, respectively, as seen in Fig. 1c. Nevertheless, the results mentioned later confirm that the remaining depth of the traps is still deep enough to trap individual or cluster of the charged microparticles. In our device, as the medium is de-ionized (D.I.) water, the Debye length is in the order of $1 \mu \mathrm{m}$, which is deduced from the equation: $\lambda_{\mathrm{D}}=\left(8 \pi l_{\mathrm{B}} c_{\infty}\right)^{-1 / 2}$. Here $l_{\mathrm{B}}$ is Bjerrum length in water $\left(0.71 \mathrm{~nm}\right.$ for univalent ion) and $c_{\infty}$ is salt ion concentration ( $10^{-7} \mathrm{M}$ in D.I. water).

\subsection{Device Fabrication}

Figure $2 \mathrm{a}$ is a 3D illustration of the whole device. The whole device was fabricated on a piece of glass substrate. The metallic stripes of the electrostatic traps and the channel are separated by a dielectric layer (SOG in the illustration). Figure $2 \mathrm{~b}$ is a front view of Fig. 2a through the channel, showing the structure and materials of the device. Note that our traps are not truly 3D. However, the limited height $h$ of the fluidic channel sets the maximum $z$ (see definition in Fig. 1) for micro-particles moving inside the channel and therefore allows the electrostatic trap to work properly. Figure 3 is an optical photograph of an actual fabricated device in vertical view. 


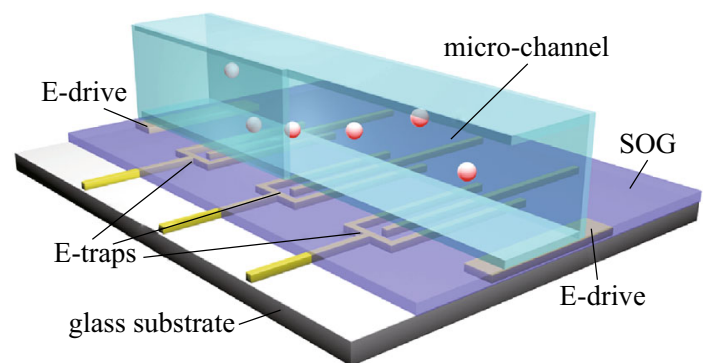

(a)

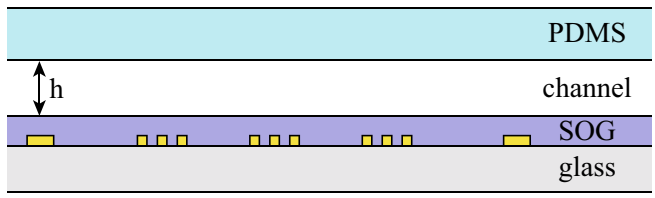

(b)

Fig. 2 Illustration of the structure and materials for the electrostatic micro-tweezers. a A $3 \mathrm{D}$ view of the electrostatic micro-tweezers in a microfluid, which includes driving electrodes, trapping electrodes, and the microfluidic channel. b Front view of the device showing the structure and materials

A set of driving electrodes, marked as $E$-drive in Figs. 2 and 3 , offers an electric field in the desired direction to drive target particles in the microchannel. When the target particles move into the trapping region (the region between two outer parallel metallic electrodes in one electrostatic trap, marked by the blue box in the inset of Fig. 3), an external static voltage is supplied to the electrode stripes, which activates the trap and keeps the particles captured. Turning off of the external static voltage deactivates the trap and the target particles will be driven out of the trapping region quickly under the driving field.

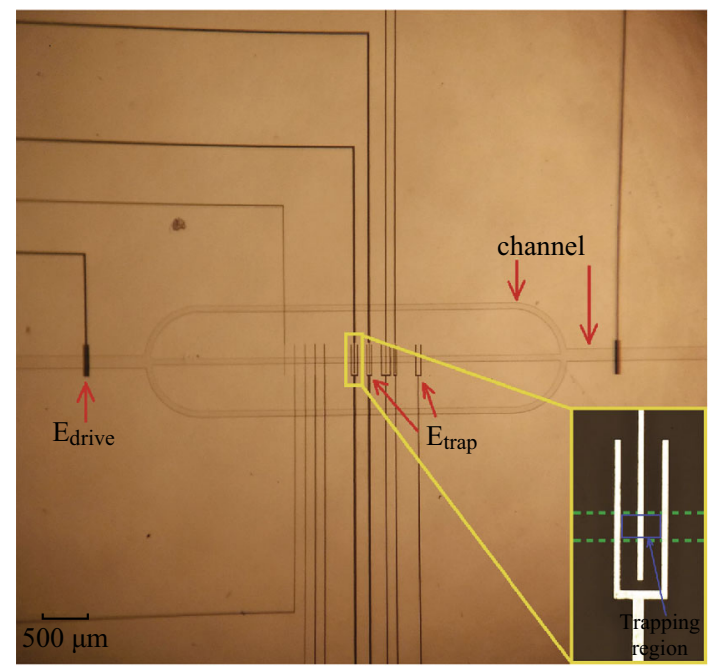

Fig. 3 Photograph of the electrostatic micro-tweezers. The inset shows the enlarged images of the trap, where the location of the fluidic channel is highlighted with dashed green lines. (Color figure online)
The electrode substrates were fabricated with standard micro-electro-mechanical system (MEMS) techniques in a clean room. Briefly, as schematically shown in Fig. 2b, a Ti/ $\mathrm{Au}$ film $(5 / 100 \mathrm{~nm})$ was patterned on a $4^{\prime \prime}$ glass wafer by photolithography (Instrument: SUSS, MBJ4), coating process (Instrument: Kurt J. Lesker, PVD 75), and lift-off process to function as the electrodes [59]. Then, a 300-nm-thick spin-on-glass (SOG) (Futurrex, Inc, China) was spin-coated on the wafer as the dielectric layer. The fluidic channel was prepared by traditional polydimethylsiloxane (PDMS)based soft lithography approach. PDMS (DOW CORNING, China) pre-polymer (curing agent: base $=1: 10$ ) was poured onto a micro-fabricated master (silicon wafer etched by deep reaction ion etching, DRIE) with designed feature sizes, and cured at $70{ }^{\circ} \mathrm{C}$ for $1 \mathrm{~h}$. Afterwards, the PDMS was peeled off from the master and holes were punched to form the inlet/ outlet. Finally, the PDMS microchannel was bonded with the electrode substrate after oxygen plasma treatment for $50 \mathrm{~s}$. After the microscale processing, the device was bonded to a Printed Circuit Board for convenient manipulation and measurement.

Microscope (NOVEL) and Color Video Camera (JVC:TK-C9211EC) were used for observing the motion of micro-particles in the fluidic channels and for recording videos. As shown in Fig. 1, the microscope observation was taken along the $z$ direction, i.e., for motion of micro-particles in a 3D space (in the fluidic channel), and all the recorded images and videos were indeed projections of the motion in the $x-y$ plane. In order to identify the transparent polystyrene (PS) particles in the microchannel, the asymmetric light beam from microscope was irradiated on the channel.

\subsection{Surface Charge Density of Micro-particles}

Two kinds of micro-particles have been applied in the experiments, namely $1-\mu \mathrm{m}$-diameter negatively charged $\mathrm{SiO}_{2}$ particles (Baseline, China) and 5- $\mu$ m-diameter polystyrene (PS) particles (Baseline, China) which exhibit positive charge due to surface-bound amino groups. Figure $4 \mathrm{a}, \mathrm{b}$ shows their scanning electron microscopy (SEM) micrographs, respectively.

In a first step, the surface charge densities of these micro-particles were determined. The particles were suspended in D.I. water and their motion under directional driving electrostatic fields from zero up to $1000 \mathrm{~V} \mathrm{~m}^{-1}$ has been observed. The velocity of each individual microparticle was measured directly by comparing its position in the video frames taken at different points in time. Figure $4 \mathrm{c}$ plots measurement data from 33 PS micro-particles. A linear fit of the data gives a gradient $\sim 3 \times 10^{-8} \mathrm{~m}^{2}$ $(\mathrm{V} \mathrm{s})^{-1}$. Note that the fit line does not tend to zero due to the existence of viscous drag, which delays the movements of the particles until the driving force is large enough. After 


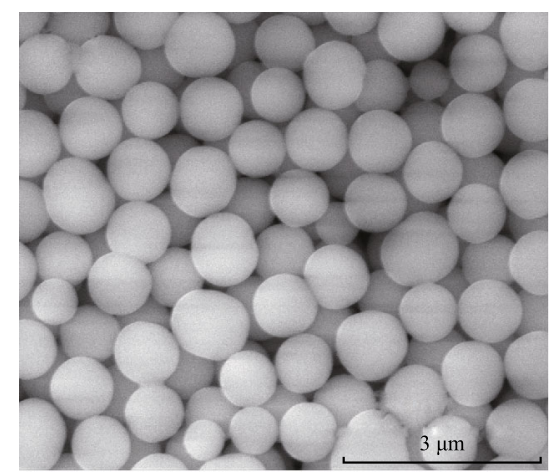

(a)

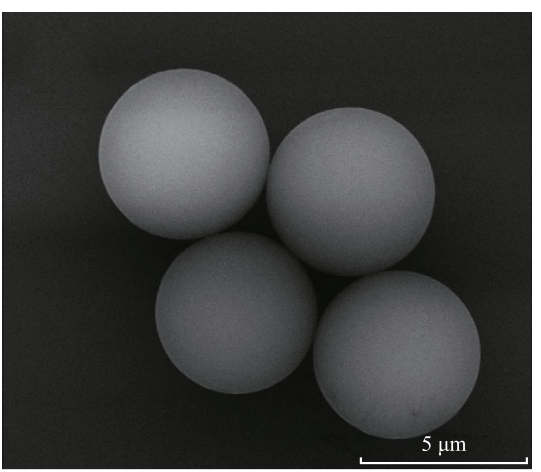

(b)

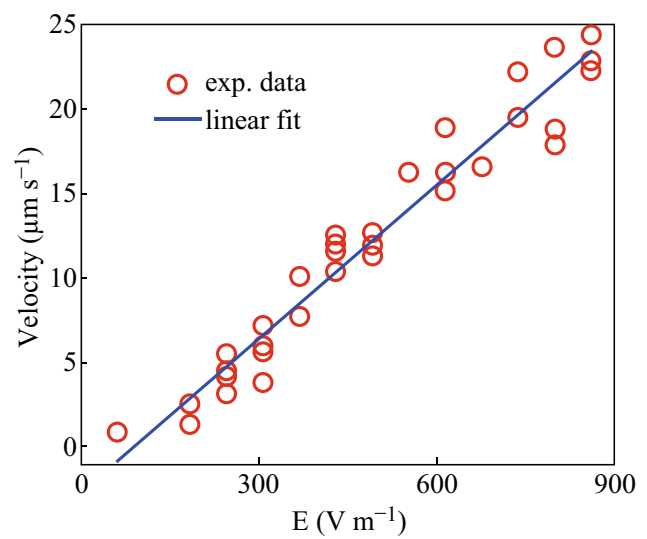

(c)

Fig. 4 Characterization of charged micro-particles used in the experiments. a, b SEM micrographs of $\mathrm{SiO}_{2}$ and polystyrene particles, showing an average diameter around $1 \mu \mathrm{m}$ and near $5 \mu \mathrm{m}$, respectively. $\mathbf{c}$ The red circles are measurement results of the velocity versus applied driving electric field in the fluid for 33 individual 5- $\mu \mathrm{m}$-diameter PS particles. The blue line is a linear fit. (Color figure online)

achieving equilibrium, the motion of the micro-particles follows the Stokes Law, $f=6 \pi r \eta v$, where $f$ is the viscous drag $(\approx$ driving force at equilibrium), $r$ the radius of the particle, $\eta$ the fluid viscosity, and $v$ the particle velocity. Here the driving force (Coulomb force) $f$ approximately fulfills $f=q E=q U / d$, where $d$ and $U$ are the distance between two electrodes and the applied voltage, respectively. From the two equations above, taking $\eta$ as 890 $\mu \mathrm{Pa} \mathrm{s}$, an average surface charge density of $1.1 \times 10^{-4}$ $e \mathrm{~nm}^{-2}$ is derived for the 5- $\mu \mathrm{m}$-diameter PS particles (where $e$ is the elementary charge with a value of $\sim 1.6 \times 10^{-19} \mathrm{C}$ ). Similarly, the average surface charge density for the 1 - $\mu$ m-diameter $\mathrm{SiO}_{2}$ micro-particles was calculated to be $2.7 \times 10^{-4} e \mathrm{~nm}^{-2}$ (data not shown). It has to be mentioned that the surface charge density of the micro-particles studied in this work is about 3-4 orders of magnitude lower than that observed in some biomacromolecules.

The $\mathrm{SiO}_{2}$ micro-particles do exhibit a $\sim 2.5$ times higher net charge density than the PS particles. As the surface area of the PS particles is $\sim 25$ times larger, each PS particle roughly carries 10 times more charges than an $\mathrm{SiO}_{2}$ particle. Thus, a total particle charge of $\sim 7900 e$ (PS) and $\sim-830 e\left(\mathrm{SiO}_{2}\right)$ can be calculated.

\section{Results}

\subsection{Driving Micro-particles in a Microchannel}

With the help of an external field, charged micro-particles could be driven inside the microchannels, as shown in Fig. 5 (see Video 1 in supplemental file). Here negatively charged $\mathrm{SiO}_{2}$ particles, appearing as bright white dots in the video, do not show any direct movement when no external field is applied, as shown in Fig. 5a. Once a DC voltage is applied with the field direction from left to right (highlighted with red arrows), the negatively charged $\mathrm{SiO}_{2}$ particles start to move from right to left in the microchannel as shown in Fig. 5a-c. Upon reversing the direction of the driving field, the particles then move backwards from left to right, as shown in Fig. 5d-f. It has 

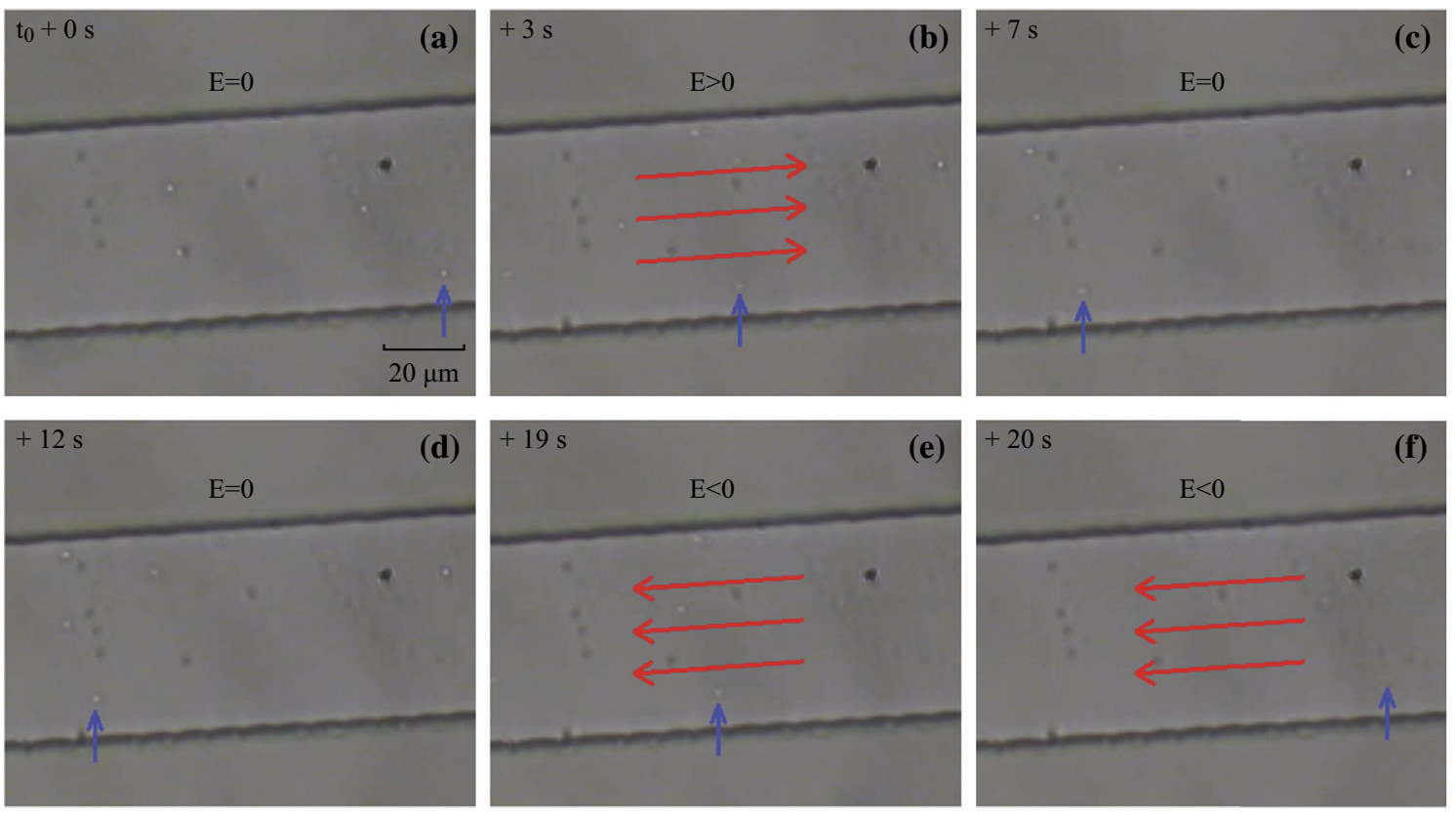

Fig. 5 Video frames showing device's manipulation for driving negatively charged $\mathrm{SiO}_{2}$ directly. a-c The particle indicated by a blue arrow is driven from the right side to the left when applying electric field towards right. c, $\mathbf{d}$ The particle stops and takes Brownian Motion when turning off the applied voltage. $\mathbf{d}-\mathbf{f}$ The particle moves backwards when changing the direction of applied electric field, and the velocity increases with the driving voltage. (Color figure online)

to be mentioned that the moving velocity of these particles can be controlled by the driving field intensity. After the driving field is switched off, the particles return to a stationary status with observable Brownian motion.

\subsection{Capture of Individual Micro-particles}

Capture of individual charged micro-particles in a controllable way is the key function of electrostatic traps. Suspensions of individual charged PS and $\mathrm{SiO}_{2}$ particles were used to verify the controllability and effectiveness of the device, as shown in Figs. 6 and 7.

Figure 6 shows a set of video frames (see Video 2 in supplemental file) demonstrating the real-time observation of PS micro-particles in a 3-stripe trapping device. As the original video has a sampling speed of 25 frames per second, the position of individual PS particles at a certain point in time could be determined. The starting time of the first frame is set as $t_{0}$, and the time points for consequent frames are determined by the number of frames in between, utilizing a constant time interval of $40 \mathrm{~ms}$ between adjacent frames. This time point is then highlighted on the upper left corner of each frame.

In Fig. 6a, the particle marked with a purple arrow is a PS micro-particle firmly adsorbed on the wall of the channel, and therefore does not move in the whole observation period. The time $t_{0}$ is chosen when a moving PS particle (highlighted with a blue arrow) occurs in the view of the CCD video camera. No excitation voltage is applied to the 3-stripe electrostatic trap, so the PS particle passes through the trapping region with a constant velocity $\left(\sim 39 \mu \mathrm{m} \mathrm{s}^{-1}\right)$, under the driving electric field.

At the time $t=t_{0}+4.80 \mathrm{~s}$, a second PS particle (indicated by a red arrow) appears in the observation region (Fig. 6c), moving at a constant velocity similar to the first one. When the second particle moves into the trapping region, which is roughly situated within the area confined by the two inner edges of the positively charged metallic stripes (Fig. 3), at $t \approx t_{0}+6.00 \mathrm{~s}$, a DC voltage of $2 \mathrm{~V}$ is applied to the 3 electrodes by manual control, activating the electrostatic potential well. Immediately, the second particle is captured near the central electrode. After a while, the trap is deactivated $\left(t \approx t_{0}+8.00 \mathrm{~s}\right)$, releasing the particle from the trap. Subsequently, the particles started to move under the driving electric field again.

During the period when the second PS particle is trapped (from $t=t_{0}+6.64 \mathrm{~s}$ to $t=t_{0}+8.12 \mathrm{~s}$ ), the particle is not fully stopped. Indeed, one sees that it is vibrating and oscillating at the original trapped location.

After reversing the voltage applied on the trap electrodes, the negatively charged $\mathrm{SiO}_{2}$ particles can be captured by the same device. This shows the flexibility of such electrically controlled electrostatic trap. Figure 7 shows a series of 8 video frames (see Video 3 in supplemental file), illustrating the motion of $1-\mu \mathrm{m}$-diameter $\mathrm{SiO}_{2}$ particles inside the microchannel. Here $t_{0}$ is again set at the point 

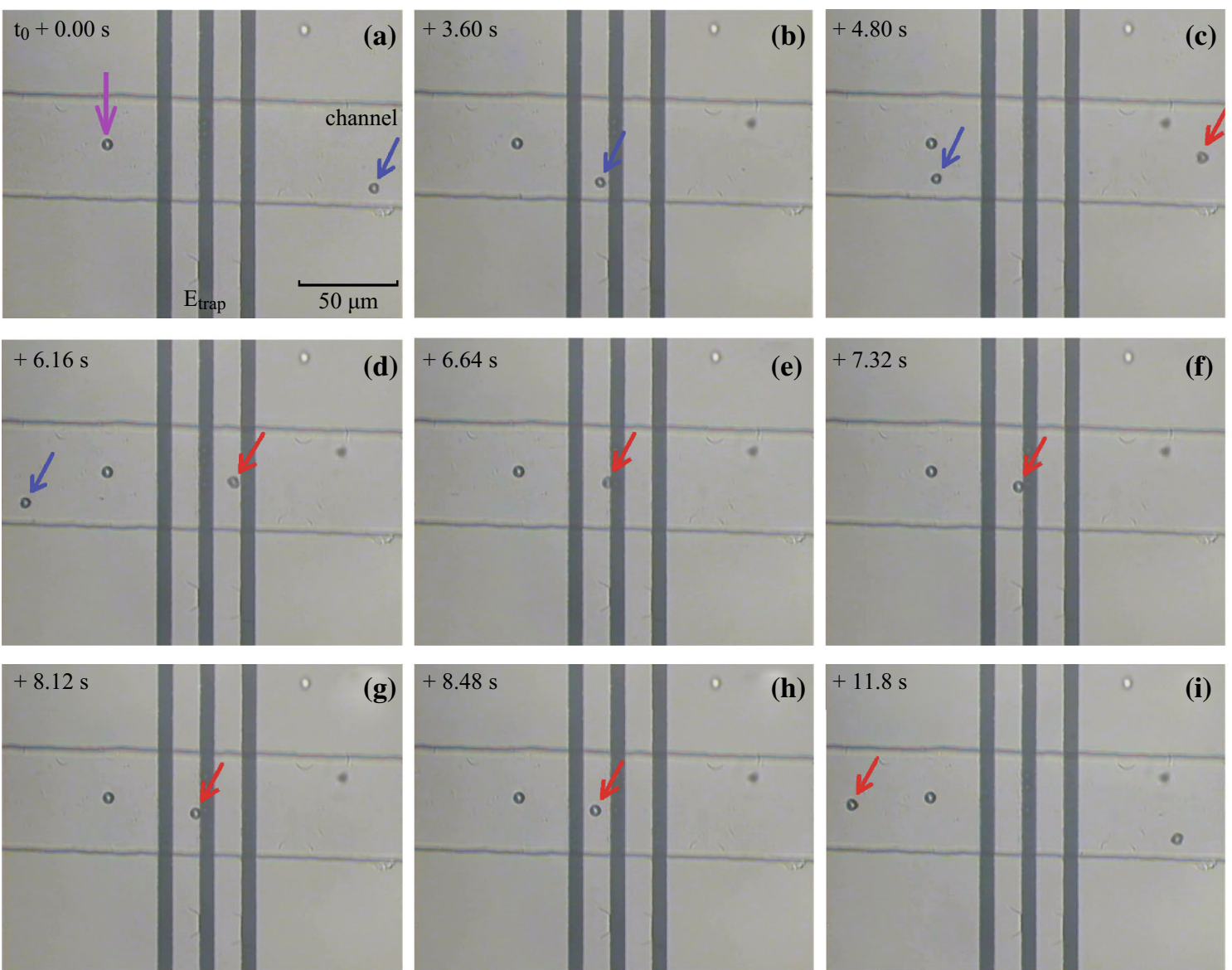

Fig. 6 Video frames showing capture of a single PS particle with a 3-stripe electrostatic trap. The nine photo images are taken from a movie, which records the functions of trapping and releasing individual PS particles, which are positively charged. A constant driving electric field is applied in parallel to the microchannel from right to left. The number marked on the upper left corner of each frame indicates the time when the frame is taken as compared to the first one. a-c The trap is not excited, so the particle marked with a blue arrow moves freely across the trapping region. $\mathbf{d}-\mathbf{g}$ The trap is turned on, and a PS particle marked with a red arrow is trapped near the central electrode. $\mathbf{h}, \mathbf{i}$ The trap is turned off, thus the trapped particle quickly leaves the trapping region. (Color figure online)
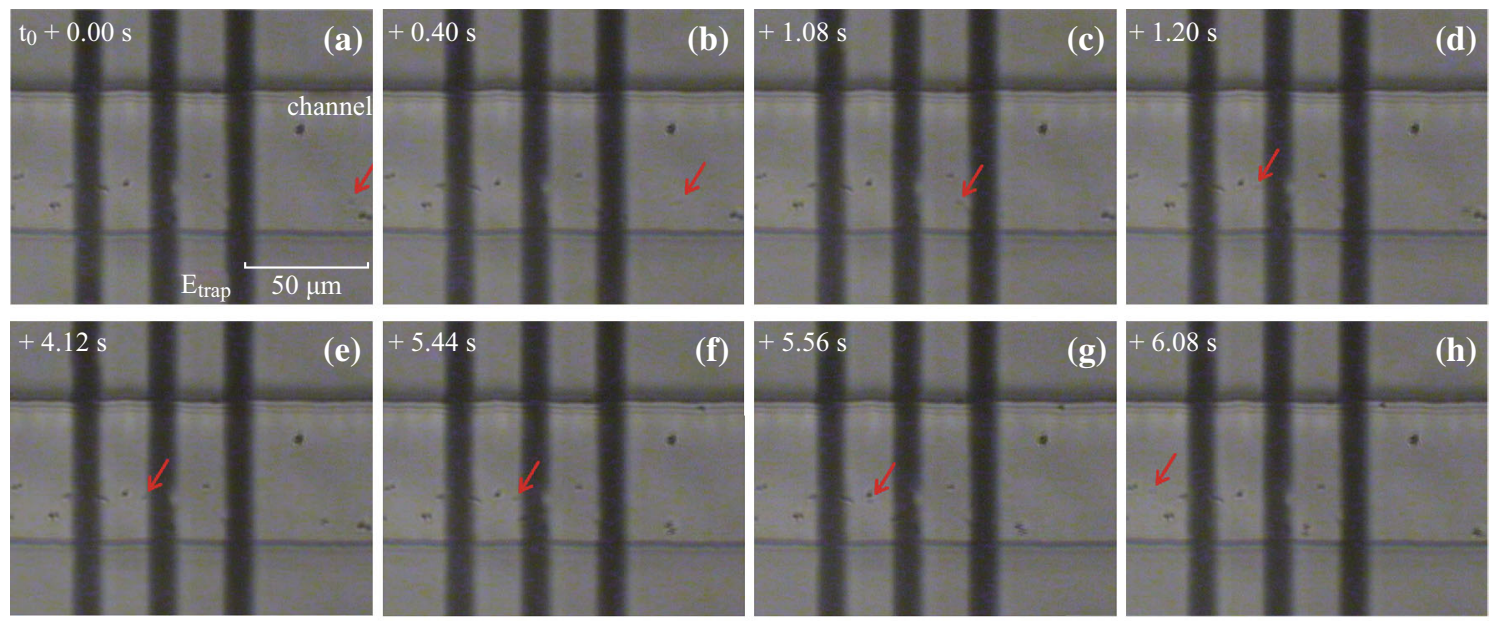

Fig. 7 Video frames showing trapping and releasing operation for an individual $\mathrm{SiO}_{2}$ micro-particle (marked with a red arrow) with a 3-stripe electrostatic trap. (Color figure online) 

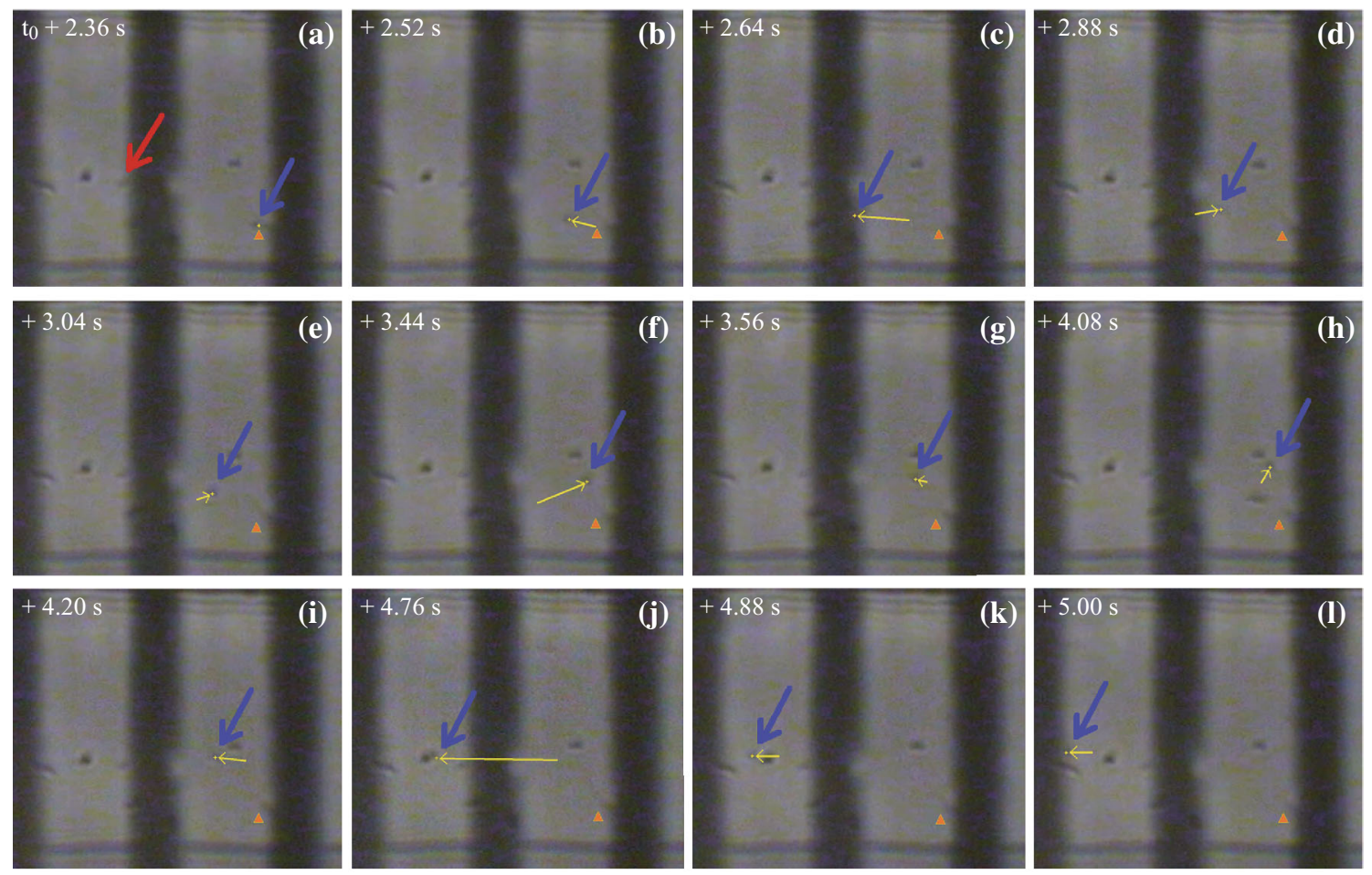

Fig. 8 A set of photos showing the motion track of another $\mathrm{SiO}_{2}$ particle (marked with a blue arrow) while the first particle is kept trapped. The initial location of the second particle is marked with an orange triangle, and each move of this particle from its previous position is highlighted with a yellow line. (Color figure online)

when an $\mathrm{SiO}_{2}$ particle, indicated by the red arrow in Fig. $7 \mathrm{a}$, occurs under a small driving electric field. The particle moves quickly from the right to the left in the observation area. At $t \approx t_{0}+1.00 \mathrm{~s}$, the trap is activated and then captures the particle at the central region of the trap (the minimum region of the Coulomb potential well). The particle remains in the trap until the trap is deactivated manually at $t \approx t_{0}+5.00 \mathrm{~s}$. Afterwards, it moves out of the trapping region along the original direction, as shown in Fig. 7f-h.

The 12 frames in Fig. 8 are magnified images of the trapping region shown in Fig. 7, and describe the motion of another $\mathrm{SiO}_{2}$ particle, exhibiting an interesting phenomenon. In the period from $t \approx t_{0}+2.36 \mathrm{~s}$ to $t \approx t_{0}+5.00 \mathrm{~s}$ when the $\mathrm{SiO}_{2}$ particle mentioned in Fig. 7 is kept trapped near the central electrode, another $\mathrm{SiO}_{2}$ particle (marked by the blue arrow) is moving inside the trap. It is previously adsorbed to the right electrode before the trap is activated. However, this $\mathrm{SiO}_{2}$ particle starts to move at $t \approx t_{0}+2.36 \mathrm{~s}$, moving leftwards or rightwards within the trapping region. A small yellow dot is used to highlight the center of this moving particle, and the location of its first appearance in the view region is marked with an orange triangle. For each two successive frames, the shift of the particle location is highlighted with a yellow vector. The behavior of this particle indicates that a sufficient trapping region exists between the two outer electrodes, which is consistent with our simulation results in Fig. 1.

\subsection{Capture of Individual Micro-particle Clusters}

The device shows the potential of trapping not only individual micro-particles, but also micro-particle clusters, as shown in Fig. 9. Here a cluster of $\mathrm{SiO}_{2}$ particles is naturally formed in the fluidic microchannel, and captured by a 3 -stripe electrostatic trap. The cluster has the shape of an asymmetric chain, so when it rotates randomly in the fluid, probably due to Brownian motion, the shape of its projection in the microscopy images (i.e., in the $x-y$ plane, see definition in Fig. 1) keeps on changing.

The 12 frames displayed in Fig. 9 are taken from a 5.16s-long video (see Video 4 in supplemental file), whereas $t_{0}$ is the time when the micro-particle cluster (indicated by the red arrow) appears in the visual field of the CCD camera. The negatively charged cluster is driven from the right towards the left by a constant electrical field. The electrostatic trap is turned on at $t=t_{0}+1.20 \mathrm{~s}$, when the cluster just moves into the region of Coulomb potential well. After the trap is turned off at $t=t_{0}+4.48 \mathrm{~s}$, the cluster starts to move out of the trapping region.

The inset in each frame in Fig. 9 is an enlarged image of the marked yellow region showing the shape of the cluster 

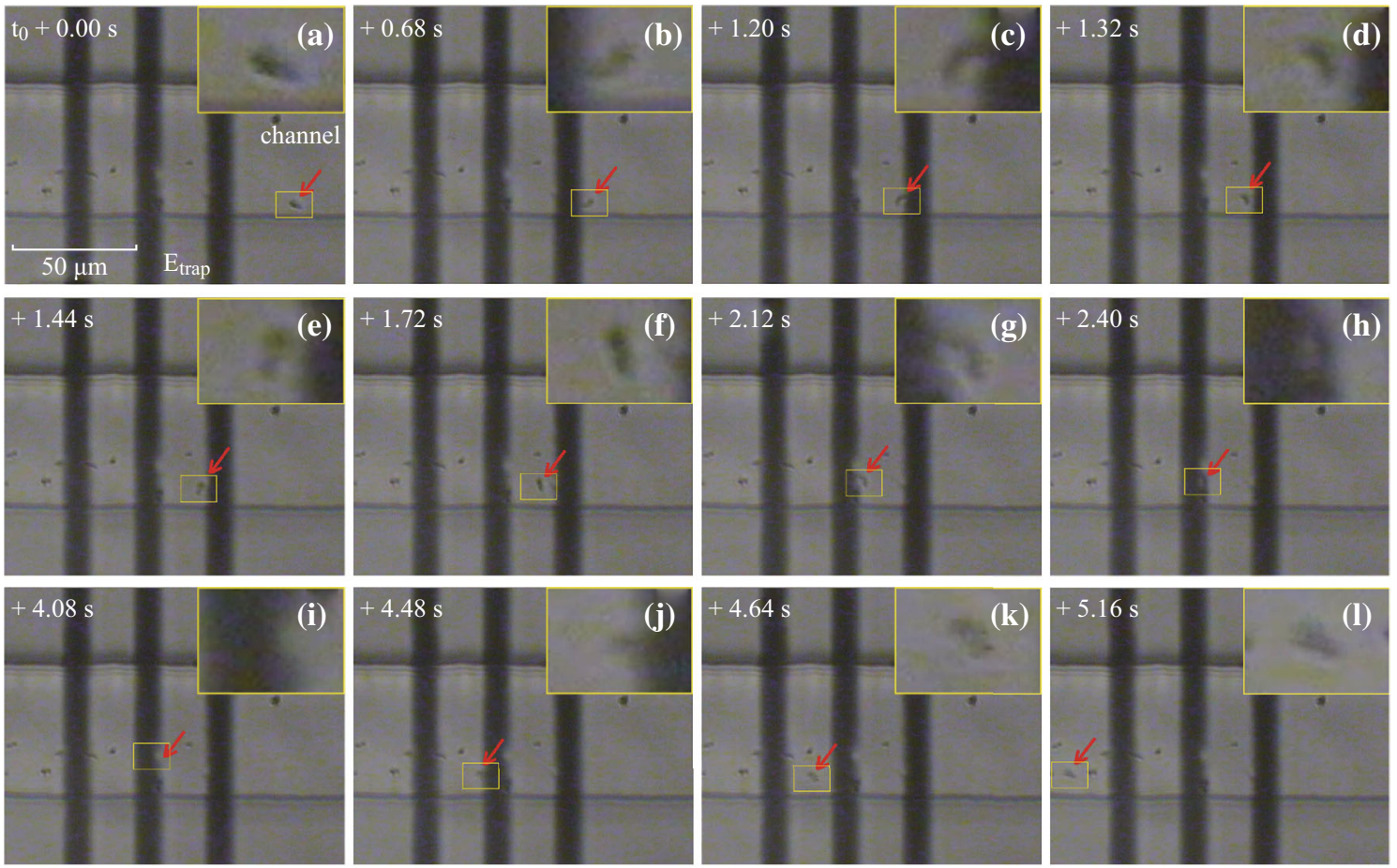

Fig. 9 Video frames showing capture of a cluster of $\mathrm{SiO}_{2}$ micro-particles with a 3-stripe electrostatic trap. a, b The trap is in an idle state, and the cluster is moving freely under an electrical driving force. $\mathbf{c}-\mathbf{j}$ The trap is turned on, and the cluster is shaking, rotating, and moving slightly in the trap. $\mathbf{k}$, $\mathbf{l}$ The trap is tuned off, and the cluster moves out of the trapping region. The inset of each frame is an enlarged image of the cluster in the region marked with a yellow rectangle, showing roughly the 3D orientation of the cluster in the fluid. (Color figure online)

in detail. One sees that during the period from $t=t_{0}+1.32 \mathrm{~s}$ to $t=t_{0}+2.40 \mathrm{~s}$, the trapped cluster is still in a status of slow motion. It changes position in the trap and meanwhile rotates randomly. These are the typical features showing the nature of Coulomb potential well designed in this work.

\section{Discussion}

As illustrated in Fig. 1, the Coulomb potential well used in the present device as ESTs has a wide trapping region in the $x-y$ plane, and a particle being trapped may find local energy minimum locations. Several physical mechanisms of a microfluid under electrical fields, such as Brownian motion, screen effect, electroosmosis, and thermophoresis, may influence the trapping performance. The method described in this work is similar to DEP technique, but still there is an essential difference between these two methods.

\subsection{Effect of Brownian Motion}

Our simulation results show that, with an excitation voltage of $1 \mathrm{~V}$, the corresponding depth of the Coulomb potential well for a micro-particle carrying a net charge of $1000 e$ is much higher than the kinetic energy (in the order of $0.1 \mathrm{meV}$ ). Thermal energy $k_{\mathrm{B}} T$, which is $26 \mathrm{meV}$ at room temperature, plays an important role when trapping nanosized particles [60, 61]. But for trapping micro-particles in the current work, the thermal energy is the measure from fluctuation of thermal motion of a vast number of molecules, atoms, ions, and clusters in the solution. These fluctuations do not add up to a net force which could induce directional movement of the micro-particles. They only result in Brownian motion, which has an extremely low kinetic energy compared to the trap depth in energy. Therefore, the thermal energy is not a key issue of concern.

\subsection{Screen Effect}

The screen effect is a common phenomenon in a microfluidic system. It is mainly caused by the adsorption of ions at the liquid-solid interface [62]. The screen effect remarkably reduces the effective surface charge density at the trap and driving electrodes. As a result, the effective field generated by the driving electrodes is reduced, thus the real average charge each micro-particle carries should be higher than what we have calculated. The curve of potential depth versus height in Fig. 1c has taken the screen effect on trap electrodes rather than the screen effect on 
particles into consideration; the latter could weaken the trap's constraint on charged particles.

\subsection{Effects of Electroosmosis and Thermophoresis}

Electroosmosis is another common phenomenon in a microfluid system, which is mainly determined by the $\zeta$ potential of the electrical double layer (EDL) and the geometric configuration of the electrodes. In our devices, as the spacing of two counterpart electrodes is only $20 \mu \mathrm{m}$, when applying a DC voltage of $1-2 \mathrm{~V}$ on the trapping electrodes, a large electric field is generated in the microfluidic channel and vortexes may therefore occur in the trapping region [63, 64]. The micro-particles being trapped could receive additional kinetic energy from the electroosmosis flow. Compared to the depth of the Coulomb potential well of the device, this effect induces only vibration, rotation, and drifting of the trapped particles inside the trapping region, as shown in Videos 1-4 in supplemental files. But the effect could not release the particle from the trap as the electroosmosis flow in D.I. water is weak.

Furthermore, a weak electroosmosis current causes little joule heat, thus not inducing a remarkable increase of the local temperature in the fluid [65]. In other words, the thermophoresis effect is also weak and would not severely affect the trapping performance.

\subsection{Similarity and Difference Between the Current Method and DEP Technique}

The DEP technique also employs local electrodes with desired patterns. In DEP, non-uniform electric fields, usually generated with an AC power [21, 66], are utilized to trap dielectric particles (either charged or not) $[67,68]$, and the flow of microfluidics is used to drive the particles [66]. DEP has been applied in high-throughput aggregation and sorting of particles or bio-particles with different characteristics including size, dielectric properties, and bioactivity [69]. However, EST may show the merit of trapping and manipulation of individual nano/micro-particles in certain applications. It is feasible to combine both EST and DEP in one system to trap and manipulate target particles or biomolecules effectively and precisely.

\section{Conclusion}

In summary, we have demonstrated basic concepts of ESTs, i.e., driving, trapping, and releasing charged micro-particles, with trapping and driving electrodes embedded in a microfluidic device. The surface charge density of the trapping electrodes and the status of the traps, as well as the velocity and direction of the micro-particles, are controlled individually with external DC voltages. As the motion of micro-particles in the microchannel was limited by the height of the fluid channel, the local Coulomb potential well formed by a 3-stripe electrostatic trap cannot be fully considered 3D. Nevertheless, the whole device worked well in the trapping performance for positively charged 5 - $\mu \mathrm{m}$-diameter PS particles and negatively charged 1 - $\mu$ m-diameter $\mathrm{SiO}_{2}$ particles. The results showed that the devices could drive, trap, and release individual micro-particles and/or clusters, making it a promising technique for analysis of single micrometer-sized subjects. Motions of random vibration, drifting, and rotation of trapped particles (clusters) indicated clear characteristics of electrostatic traps. Other effects including Brownian Motion, screen effect, electroosmosis, and thermophoresis were discussed, and seem to have noncritical influences on the trapping performance.

The performance of the presented devices can be further improved by the addition of sensing of target objects, or by the usage of more sophisticated electrode structures (e.g., a multi-ring configuration). The $3 \mathrm{D}$ shape of the Coulomb well can also be adjusted with a precise control of net charge density distribution on the trapping electrodes, e.g., by fixing earth lines in the microchannels. We believe that the working principle of the current device is applicable to smaller devices; therefore, it may lead to a sophisticated technique of "electrostatic nano-tweezers" for sorting, separation, trapping, and manipulation of individual charged nanoparticles, such as proteins and DNA, which are often naturally charged.

Acknowledgments We thank Yaoping Liu, Qian Sheng, Jianming Xue, Martin Gose, and Xiaoye Huo for valuable discussions. We thank Mengmeng Xiao for assistance in SEM characterization of the micro-particles. This work is financially supported by National Natural Science Foundation of China (NSFC Grants No. 11374016) and MOST of China (Grant 2012CB932702, 2011CB933002).

Open Access This article is distributed under the terms of the Creative Commons Attribution 4.0 International License (http://creative commons.org/licenses/by/4.0/), which permits unrestricted use, distribution, and reproduction in any medium, provided you give appropriate credit to the original author(s) and the source, provide a link to the Creative Commons license, and indicate if changes were made.

\section{References}

1. F. Dalfovo, S. Giorgini, L.P. Pitaevskii, S. Stringari, Theory of Bose-Einstein condensation in trapped gases. Rev. Mod. Phys. 71(3), 463-512 (1999). doi:10.1103/RevModPhys.71.463

2. M.H. Anderson, J.R. Ensher, M.R. Matthews, C.E. Wieman, E.A. Cornell, Observation of bose-einstein condensation in a dilute atomic vapor. Science 269(5221), 198-201 (1995). doi:10.1126/ science. 269.5221 .198 
3. S. Chu, Cold atoms and quantum control. Nature 416(6877), 206-210 (2002). doi:10.1038/416206a

4. S.T. Cundiff, J. Ye, Colloquium: Femtosecond optical frequency combs. Rev. Mod. Phys. 75(1), 325-342 (2003). doi:10.1103/ RevModPhys.75.325

5. S.A. Diddams, J.C. Bergquist, S.R. Jefferts, C.W. Oates, Standards of time and frequency at the outset of the 21 st century. Science 306(5700), 1318-1324 (2004). doi:10.1126/science. 1102330

6. S. Chu, Laser manipulation of atoms and particles. Science 253(5022), 861-866 (1991). doi:10.1126/science.253.5022.861

7. J. Ye, H.J. Kimble, H. Katori, Quantum state engineering and precision metrology using state-insensitive light traps. Science 320(5884), 1734-1738 (2008). doi:10.1126/science.1148259

8. A. Ashkin, Optical trapping and manipulation of neutral particles using lasers. Proc. Natl. Acad. Sci. USA 94(10), 4853-4860 (1997). doi:10.1073/pnas.94.10.4853

9. W.M. Lee, X.C. Yuan, W.C. Cheong, Optical vortex beam shaping by use of highly efficient irregular spiral phase plates for optical micromanipulation. Opt. Lett. 29(15), 1796-1798 (2004). doi:10.1364/ol.29.001796

10. S. Sato, H. Inaba, Optical trapping and manipulation of microscopic particles and biological cells by laser beams. Opt. Quant. Electron. 28(1), 1-16 (1996). doi:10.1007/bf00578546

11. M. Andersson, O. Axner, F. Almqvist, B.E. Uhlin, E. Fallman, Physical properties of biopolymers assessed by optical tweezers: Analysis of folding and refolding of bacterial pili. ChemPhysChem 9(2), 221-235 (2008). doi:10.1002/cphc.200700389

12. K. Norregaard, L. Jauffred, K. Berg-Sorensen, L.B. Oddershede, Optical manipulation of single molecules in the living cell. Phys. Chem. Chem. Phys. 16(25), 12614-12624 (2014). doi:10.1039/ c4cp00208c

13. S. Seeger, S. Monajembashi, K.J. Hutter, G. Futterman, J. Wolfrum, K.O. Greulich, Application of laser optical tweezers in immunology and molecular-genetics. Cytometry 12(6), 497-504 (1991). doi:10.1002/cyto.990120606

14. P. Ben-Abdallah, A.O. El Moctar, B. Ni, N. Aubry, P. Singh, Optical manipulation of neutral nanoparticles suspended in a microfluidic channel. J. Appl. Phys. 99(9), 094303 (2006). doi:10. $1063 / 1.2191572$

15. W.H. Zhang, L.N. Huang, C. Santschi, O.J.F. Martin, Trapping and sensing $10 \mathrm{~nm}$ metal nanoparticles using plasmonic dipole antennas. Nano Lett. 10(3), 1006-1011 (2010). doi:10.1021/ nl904168f

16. S. Dash, S. Mohanty, Dielectrophoretic separation of micron and submicron particles: a review. Electrophoresis 35(18), 2656-2672 (2014). doi:10.1002/elps.201400084

17. N. Lewpiriyawong, C. Yang, AC-dielectrophoretic characterization and separation of submicron and micron particles using sidewall AgPDMS electrodes. Biomicrofluidics 6(1), 012807 (2012). doi:10.1063/1.3682049

18. N. Markarian, M. Yeksel, B. Khusid, K.R. Farmer, A. Acrivos, Particle motions and segregation in dielectrophoretic microfluidics. J. Appl. Phys. 94(6), 4160-4169 (2003). doi:10.1063/1.1600845

19. H.A. Pohl, I. Hawk, Separation of living and dead cells by dielectrophoresis. Science 152(3722), 647-649 (1966). doi:10. 1126/science. 152.3722 .647 -a

20. I.F. Cheng, H.-C. Chang, D. Hou, H.-C. Chang, An integrated dielectrophoretic chip for continuous bioparticle filtering, focusing, sorting, trapping, and detecting. Biomicrofluidics 1(2), 021503 (2007). doi:10.1063/1.2723669

21. R. Pethig, Review article-dielectrophoresis: status of the theory, technology, and applications. Biomicrofluidics 4, 022811 (2010). doi:10.1063/1.3456626

22. C. Zhang, K. Khoshmanesh, A. Mitchell, K. Kalantar-zadeh, Dielectrophoresis for manipulation of micro/nano particles in microfluidic systems. Anal. Bioanal. Chem. 396(1), 401-420 (2010). doi:10.1007/s00216-009-2922-6

23. W. Paul, Electromagnetic traps for charged and neutral particles. Rev. Mod. Phys. 62(3), 531-540 (1990). doi:10.1103/RevMod Phys.62.531

24. N.D. Scielzo, R.M. Yee, P.F. Bertone, F. Buchinger, S.A. Caldwell et al., A novel approach to beta-delayed neutron spectroscopy using the beta-decay Paul trap. Nucl. Data Sheets 120, 70-73 (2014). doi:10.1016/j.nds.2014.07.009

25. Y. Rondelez, G. Tresset, T. Nakashima, Y. Kato-Yamada, H. Fujita, S. Takeuchi, H. Noji, Highly coupled ATP synthesis by F-1-ATPase single molecules. Nature 433(7027), 773-777 (2005). doi:10.1038/nature03277

26. I. De Vlaminck, C. Dekker, Recent advances in magnetic tweezers. Annu. Rev. Biophys. 41, 453-472 (2012). doi:10.1146/ annurev-biophys-122311-100544

27. C. Liu, T. Stakenborg, S. Peeters, L. Lagae, Cell manipulation with magnetic particles toward microfluidic cytometry. J. Appl. Phys. 105, 102014 (2009). doi:10.1063/1.3116091

28. C. Bao, L. Chen, T. Wang, C. Lei, F. Tian, D. Cui, Y. Zhou, One step quick detection of cancer cell surface marker by integrated NiFe-based magnetic biosensing cell cultural chip. Nano-Micro Lett. 5(3), 213-222 (2013). doi:10.5101/nml.v5i3.p213-222

29. L. Yu, H. Wu, B. Wu, Z. Wang, H. Cao, C. Fu, N. Jia, Magnetic $\mathrm{Fe}_{3} \mathrm{O}_{4}$-reduced graphene oxide nanocomposites-based electrochemical biosensing. Nano-Micro Lett. 6(3), 258-267 (2014). doi: $10.5101 / \mathrm{nml} 140028 \mathrm{a}$

30. T.R. Strick, G. Charvin, N.H. Dekker, J.F. Allemand, D. Bensimon, V. Croquette, Tracking enzymatic steps of DNA topoisomerases using single-molecule micromanipulation. C. R. Phys. 3(5), 595-618 (2002). doi:10.1016/s1631-0705(02)01347-6

31. S.Y. Xu, W.Q. Sun, M. Zhang, J. Xu, L.M. Peng, Transmission electron microscope observation of a freestanding nanocrystal in a Coulomb potential well. Nanoscale 2(2), 248-253 (2010). doi:10.1039/b9nr00144a

32. V.P. Oleshko, J.M. Howe, Are electron tweezers possible? Ultramicroscopy 111(11), 1599-1606 (2011). doi:10.1016/j.ultra mic.2011.08.015

33. V.P. Oleshko, J.M. Howe, Advances in imaging and electron physics, vol. 179 (Elsevier Academic Press Inc., San Diego, 2013), pp. 203-262. doi:10.1016/b978-0-12-407700-3.00003-x

34. A.A. Kayani, K. Khoshmanesh, S.A. Ward, A. Mitchell, K. Kalantar-Zadeh, Optofluidics incorporating actively controlled micro- and nano-particles. Biomicrofluidics 6(3), 32 (2012). doi:10.1063/1.4736796

35. K. Kalantar-zadeh, J.Z. Ou, T. Daeneke, M.S. Strano, M. Pumera, S.L. Gras, Two-dimensional transition metal dichalcogenides in biosystems. Adv. Funct. Mater. 25(32), 5086-5099 (2015). doi:10.1002/adfm.201500891

36. J. Chen, D. Chen, Y. Xie, T. Yuan, X. Chen, Progress of microfluidics for biology and medicine. Nano-Micro Lett. 5(1), 66-80 (2013). doi:10.3786/nml.v5i1.p66-80

37. I. Artsimovitch, M.N. Vassylyeva, D. Svetlov, V. Svetlov, A. Perederina, N. Igarashi, N. Matsugaki, S. Wakatsuki, T.H. Tahirov, D.G. Vassylyev, Allosteric modulation of the RNA polymerase catalytic reaction is an essential component of transcription control by rifamycins. Cell 122(3), 351-363 (2005). doi:10.1016/j.cell.2005.07.014

38. S.J. Benkovic, S. Hammes-Schiffer, A perspective on enzyme catalysis. Science 301(5637), 1196-1202 (2003). doi:10.1126/ science. 1085515

39. M. Buck, W. Cannon, Specific binding of the transcription factor Sigma-54 to promoter DNA. Nature 358(6385), 422-424 (1992). doi: $10.1038 / 358422 \mathrm{a} 0$

40. F.C. Oberstrass, S.D. Auweter, M. Erat, Y. Hargous, A. Henning, P. Wenter, L. Reymond, B. Amir-Ahmady, S. Pitsch, D.L. Black, 
F.H.T. Allain, Structure of PTB bound to RNA: specific binding and implications for splicing regulation. Science 309(5743), 2054-2057 (2005). doi:10.1126/science.1114066

41. S.J. Singer, G.L. Nicolson, Fluid mosaic model of structure of cell-membranes. Science 175(4023), 720-731 (1972). doi:10. 1126/science.175.4023.720

42. T.J. Yoo, O.A. Roholt, D. Pressman, Specific binding activity of isolated light chains of antibodies. Science 157(3789), 707-709 (1967). doi:10.1126/science.157.3789.707

43. V. Zimarino, C. Wu, Induction of sequence-specific binding of drosophila heat-shoch activator protein without protein-synthesis. Nature 327(6124), 727-730 (1987). doi:10.1038/327727a0

44. F. Avbelj, J. Moult, Role of electrostatic screening in determining protein main-chain conformation preferences. Biochemistry 34(3), 755-764 (1995). doi:10.1021/bi00003a008

45. A.I. Popescu, Possible specificity of cellular interaction due to electrostatic forces. Electro- Magnetobiol. 14(2), 65-74 (1995). doi: $10.3109 / 15368379509022546$

46. A.A. Kornyshev, S. Leikin, Theory of interaction between helical molecules. J. Chem. Phys. 107(9), 3656-3674 (1997). doi:10. 1063/1.475320

47. E. Seyrek, P.L. Dubin, J. Henriksen, Nonspecific electrostatic binding characteristics of the heparin-antithrombin interaction. Biopolymers 86(3), 249-259 (2007). doi:10.1002/bip.20731

48. M.P. Singh, J. Stefko, J.A. Lumpkin, J. Rosenblatt, The effect of electrostatic charge interactions on release rate of gentamicin from collagen matrices. Pharm. Res. 12(8), 1205-1210 (1995). doi:10.1023/a:1016272212833

49. S. Sabri, A. Pierres, A.M. Benoliel, P. Bongrand, Influence of surface charges on cell adhesion: difference between static and dynamic conditions. Biochem. Cell Biol. 73(7-8), 411-420 (1995). doi:10.1139/095-048

50. I. Rouzina, V.A. Bloomfield, Competitive electrostatic binding of charged ligands to polyelectrolytes: practical approach using the non-linear Poisson-Boltzmann equation. Biophys. Chem. 64(1-3), 139-155 (1997). doi:10.1016/s0301-4622(96)02231-4

51. A.A. Kornyshev, S. Leikin, Electrostatic interaction between helical macromolecules in dense aggregates: an impetus for DNA poly- and mesomorphism. Proc. Natl. Acad. Sci. USA 95(23), 13579-13584 (1998). doi:10.1073/pnas.95.23.13579

52. U. Sharma, R.S. Negin, J.D. Carbeck, Effects of cooperativity in proton binding on the net charge of proteins in charge ladders. J. Phys. Chem. B 107(19), 4653-4666 (2003). doi:10.1021/ jp027780d

53. H.M. Berman, J. Westbrook, Z. Feng, G. Gilliland, T.N. Bhat, H. Weissig, I.N. Shindyalov, P.E. Bourne, The protein data bank. Nucleic Acids Res. 28(1), 235-242 (2000). doi:10.1093/nar/28.1. 235

54. J. Gruber, A. Zawaira, R. Saunders, C.P. Barrett, M.E.M. Noble, Computational analyses of the surface properties of protein-protein interfaces. Acta Crystallogr. D 63, 50-57 (2007). doi:10. 1107/s0907444906046762

55. P. Schaefer, D. Riccardi, Q. Cui, Reliable treatment of electrostatics in combined QM/MM simulation of macromolecules. J. Chem. Phys. 123(1), 014905 (2005). doi:10.1063/1.1940047
56. R.J. Zauhar, A. Varnek, A fast and space-efficient boundary element method for computing electrostatic and hydration effects in large molecules. J. Comput. Chem. 17(7), 864-877 (1996). doi:10.1002/(sici)1096-987x(199605)17:7<864:aid-jcc10>3.0. co;2-b

57. M. Malinska, K.N. Jarzembska, A.M. Goral, A. Kutner, K. Wozniak, P.M. Dominiak, Sunitinib: from charge-density studies to interaction with proteins. Acta Crystallogr. D 70, 1257-1270 (2014). doi:10.1107/s1399004714002351

58. M. Krishnan, N. Mojarad, P. Kukura, V. Sandoghdar, Geometryinduced electrostatic trapping of nanometric objects in a fluid. Nature 467(7316), 692-695 (2010). doi:10.1038/nature09404

59. Q.W. Zhuang, W.Q. Sun, Y.L. Zheng, J.W. Xue, H.X. Liu, M. Chen, S.Y. Xu, A multilayered microfluidic system with functions for local electrical and thermal measurements. Microfluid. Nanofluid. 12(6), 963-970 (2012). doi:10.1007/s10404-0110930-2

60. J. Koo, C. Kleinstreuer, Impact analysis of nanoparticle motion mechanisms on the thermal conductivity of nanofluids. Int. Commun. Heat Mass 32(9), 1111-1118 (2005). doi:10.1016/j. icheatmasstransfer.2005.05.014

61. W.B. Russel, Brownian-motion of small particles suspended in liquids. Annu. Rev. Fluid Mech. 13, 425-455 (1981). doi:10. 1146/annurev.fl.13.010181.002233

62. R. Tadmor, E. Hernandez-Zapata, N.H. Chen, P. Pincus, J.N. Israelachvili, Debye length and double-layer forces in polyelectrolyte solutions. Macromolecules 35(6), 2380-2388 (2002). doi: $10.1021 / \mathrm{ma} 011893 \mathrm{y}$

63. A. Ajdari, Electro-osmosis on inhomogeneously charged surfaces. Phys. Rev. Lett. 75(4), 755-758 (1995). doi:10.1103/ PhysRevLett.75.755

64. C. Kan, C. Bo, W. Jiankang, Numerical analysis of field-modulated electroosmotic flows in microchannels with arbitrary numbers and configurations of discrete electrodes. Biomed. Microdevices 12(6), 959-966 (2010). doi:10.1007/s10544-0109450-1

65. G.M. Mala, D.Q. Li, Flow characteristics of water in microtubes. Int. J. Heat Fluid Flow 20(2), 142-148 (1999). doi:10.1016/ S0142-727X(98)10043-7

66. Y. Demircan, E. Ozgur, H. Kulah, Dielectrophoresis: applications and future outlook in point of care. Electrophoresis 34(7), 1008-1027 (2013). doi:10.1002/elps.201200446

67. W. Guan, J.H. Park, P.S. Krstic, M.A. Reed, Non-vanishing ponderomotive $\mathrm{AC}$ electrophoretic effect for particle trapping. Nanotechnology 22(24), 245103 (2011). doi:10.1088/0957-4484/ 22/24/245103

68. C.-C. Chung, T. Glawdel, C.L. Ren, H.-C. Chang, Combination of ac electroosmosis and dielectrophoresis for particle manipulation on electrically-induced microscale wave structures. J. Micromech. Microeng. 25(3), 035003 (2015). doi:10.1088/09601317/25/3/035003

69. T.Z. Jubery, S.K. Srivastava, P. Dutta, Dielectrophoretic separation of bioparticles in microdevices: a review. Electrophoresis 35(5), 691-713 (2014). doi:10.1002/elps.201300424 